Relations industrielles

Industrial Relations

\title{
The Economics of Trade Unions, by Albert Rees, University of Chicago Press, 1962, pp. 202.
}

\section{Jacques St-Laurent}

Volume 17, numéro 4, octobre 1962

URI : https://id.erudit.org/iderudit/1021494ar

DOI : https://doi.org/10.7202/1021494ar

Aller au sommaire du numéro

Éditeur(s)

Département des relations industrielles de l'Université Laval

ISSN

0034-379X (imprimé)

1703-8138 (numérique)

Découvrir la revue

Citer ce compte rendu

St-Laurent, J. (1962). Compte rendu de [The Economics of Trade Unions, by Albert Rees, University of Chicago Press, 1962, pp. 202.] Relations industrielles / Industrial Relations, 17(4), 507-508. https://doi.org/10.7202/1021494ar

Tous droits réservés @ C Département des relations industrielles de l’Université Laval, 1963
Ce document est protégé par la loi sur le droit d'auteur. L’utilisation des services d'Érudit (y compris la reproduction) est assujettie à sa politique d'utilisation que vous pouvez consulter en ligne.

https://apropos.erudit.org/fr/usagers/politique-dutilisation/ 
tions à remplir qui rappellent des refrains déjà connus. Et lon sait ce que cela veut dire.

Cinq ans d'expérience syndicale dans un pays en pleine transformation économique et sociale, c'est bien peu. Mais il est intéressant de voir comment à partir d'une conception de la liberté, comme nous l'entendons dans le monde occidental, le syndicalisme est en train de s'organiser avec la sympathie du gouvernement. Ce rapport est une contribution utile à l'étude du syndicalisme et des relations industrielles dans le monde.

\section{Gérard Dion}

\section{Principes déconomie politique, par Ro-} ger Dehem, Dunod, Paris 1962, pp. 189.

Ce n'est pas tous les jours qu'un professeur de l'Université Laval publie un livre sérieux sur l'économie. C'est un évènement rare qu'il vaut la peine de signaler.

Le livre du professeur Dehem s'adresse à ceux qui s'intéressent aux grands problèmes économiques de notre temps. Il ne s'adresse pas qu'à ceux dont l'étude des phénomènes économiques est l'occupation continuelle. Tous ceux que touchent les problèmes de l'élimination de la misère o ou \&l'élimination du bien-être de la société \$ et qui veulent en amorcer une étude sérieuse auront profit à lire les « Principes d'économie politique ».

Le livre, bien qu'il soit un livre d'initiation, bien que l'auteur, pour des raisons pédagogiques, se soit abstenu d'utiliser les techniques d'analyse habituelles aux économistes, ne va pas sans difficulté. L'Auteur a échappé à la tentation de rendre trop facile ce qui ne l'est pas en risquant de perdre l'essentiel. C'est pourquoi son livre est à la fois élémentaire et approfondi. Dans ce dernier sens, c'est un livre difficile. Mais la connaissance des phénomènes économiques et des règles qui les régissent l'est aussi.

N'importe lequel lecteur se sent en terrain connu, lorsque l'auteur réfère, par exemple, à cet anglais Child comme à «un précurseur typique des plaideurs de politique économique en fonction d'intérêts particuliers 》; ou lorsqu'il rappelle que les phénomènes économiques sont liés \& non seulement aux éléments atmosphériques, mais aussi à l'échelonnement d'événements sociaux telles les fêtes de Noël et de Pâques 》. Le même lecteur se sent cependant moins à l'aise lorsque l'auteur parlera sans explication supplémentaire de $<$ la violation des conditions d'un optimum parétien $\gg$, (p. 160) ou de la démonstration faite par certains auteurs du fait que * si l'épargne pouvait être tellement abondante que sa productivité marginale descende à zéro, la «productivité sociale» de l'économie serait maxima ». (p. 159).

Mais ces passages plus difficiles devraient être pour le lecteur une invitation à un approfondissement des concepts auxquels sont habitués les économistes, lesquels concepts sont nécessaires à la compréhension des phénomènes économiques.

Cet approfondissement est rendu facile par les excellentes références qui apparaissent à la fin de chaque chapitre et qui ont le mérite d'être sûres et de n'être pas trop nombreuses.

Signalons enfin que le style est correct et concis et que le vocabulaire est moderne ( guidage », 《retombées 》, « la nouvelle vague \$, « déviation conservatrice $\gg$ ).

Le livre de M. Dehem n'est pas un livre parfait. Mais c'est un livre honnête et sérieux. Il a le mérite de ne pas éloigner les non-initiés par une surcharge de preuves géométriques ou de relations mathématiques; d'apporter aux facteurs institutionnels et politiques plus d'importance qu'en accordent les économistes; de ne pas esquiver la complexité d'une analyse qui conduit à des solutions possibles mais pas toujours certaines; de rendre évident que les problèmes économiques ne peuvent pas être résolus en disant <hocus pocus $\gg$ ou par quelques gestes de magiciens.

Nous souhaitons, avec l'auteur, que ce livre conduise à d'autres.

\section{Jacques St-Laurent}

The Economics of Trade Unions, by Albert Rees, University of Chicago Press, 1962, pp. 202.

Voici le dernier venu de l'excellente collection « Cambridge Economic Hand- 
books », édité conjointement par l'université anglaise de Cambridge et l'université de Chicago. Le but de cette collection, c'est de fournir à des lecteurs non-initiés une idée des principes que les économistes utilisent dans l'analyse des problèmes économiques contemporains. L'intérêt de cette série est donc centré sur le fait qu'elle s'adresse à des lecteurs ordinaires et qu'elle traite de problèmes courants.

Plus précisément, le livre du professeur Rees comporte principalement une discussion du rôle joué par les unions dans l'élaboration de la structure des salaires et de l'effet des changements dans cette dernière sur les prix, l'emploi, la productivité et la part des salariés dans le revenu national. Il complète de cette façon les livres connus des professeurs Ross et Dunlop et incorpore l'essentiel de la littérature parue à cet égard depuis la publication des livres de ces derniers.

Bien que ce soit l'intention de l'auteur de traiter surtout de l'aspect économique des unions ouvrières, le livre contient certains chapitres où l'on trouve l'essentiel de l'aspect institutionnel des unions. Cela permet de situer le rôle économique des unions dans son cadre institutionnel.

A cause de la qualité des lecteurs auxquels il s'adresse avant tout, l'auteur n'utilise pas les techniques d'analyse habituelles aux économistes : on ne trouvera dans le livre ni graphique, ni relations mathématiques qui risqueraient de rebuter les non-initiés. Cette absence cependant n'enlève rien à la valeur scientifique du livre. La rigueur de la démonstration est toujours apparente et l'absence d'un appareil technique ne conduit pas au verbiage. Au contraire, on remarquera la concision avec laquelle les problèmes sont discutés.

L'auteur a su éviter l'allure théorique et artificielle qui est le propre de certaines analyses économiques en illustrant ses propres références à des situations bien concrètes.

Le livre de M. Rees est certainement la présentation systématique la plus sûre, la plus complète et la plus à date du rôle économique des unions ouvrières. C'est pourquoi nous le recommandons fortement à l'attention de tous ceux qui se réjouissent ou qui s'inquiètent du rôle de cette dernière dans notre so. ciété.

\section{Jacques St-Laurent}

Labor Law; par Nicholas S. Falcone; John Wiley and Sons, Inc., New York, N.Y., 1962, 502 pp.

Le présent ouvrage se situe dans la lignée bibliographique des ouvrages consacrés au droit du travail chez nos voisins du Sud. Nous connaissions déjà les éditions de l'ouvrage de Charles $\mathbf{O}$. Gregory «Labor and the Law », le répertoire de Benjamin Werne «The Law of Industrial Relations », le traité de Glenn Miller «American Labor and the Government » ainsi que certains autres ouvrages de moindre envergure, sans compter l'immense littérature à caractère juridique ou para-juridique que les Américains ont produite depuis un certain nombre d'années en rapport avec les relations industrielles chez eux.

Une telle évolution s'explique assez bien si l'on songe au caractère fortement légaliste du système américain de relations industrielles, à l'importance et à l'universalité qu'ont acquises les règles juridiques de ce système au fur et à mesure que les pouvoirs publics sont intervenus dans ce champ d'activités au moyen de lois-cadres, de statuts particuliers, de réglementations de toutes sortes, et que les tribunaux aussi bien judiciaires qu'administratifs ont été appelés à interpréter et à appliquer une telle masse de législation.

L'ouvrage de Falcone suit une méthodologie que nous appellerions «traditionnelle », en matière de traités de droit du travail américains; il se présente toutefois selon un ordre chronologique qui permet à l'auteur de situer les grandes étapes de la législature ouvrière, d'en faire ressortir les principales caractéristiques, d'en montrer l'évolution en fonction des grands textes législatifs et des principales décisions judiciaires qui en ont informé la marche au cours des années.

Comme plusieurs autres ouvrages semblables, le présent traité contient en appendice les textes mêmes des principales lois ouvrières américaines adoptées depuis quarante ans et qui ont servi à constituer l'armature du système actuel de relations industrielles aux Etats-Unis. 